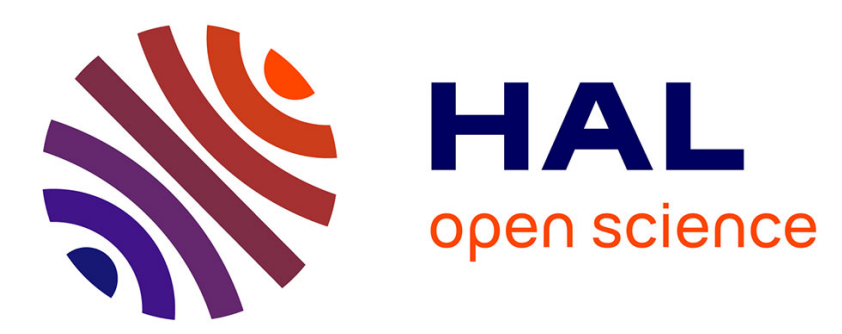

\title{
Coherent illusory contours reduce microsaccade frequency
}

\author{
Alexis. D.J. Makin, Rochelle Ackerley, Kelly Wild, Ellen Poliakoff, Emma \\ Gowen, Wael El-Deredy
}

\section{- To cite this version:}

Alexis. D.J. Makin, Rochelle Ackerley, Kelly Wild, Ellen Poliakoff, Emma Gowen, et al.. Coherent illusory contours reduce microsaccade frequency. Neuropsychologia, 2011, 49 (9), pp.2798-2801. 10.1016/j.neuropsychologia.2011.06.001 . hal-01599669

\section{HAL Id: hal-01599669 https://hal.science/hal-01599669}

Submitted on 3 Oct 2017

HAL is a multi-disciplinary open access archive for the deposit and dissemination of scientific research documents, whether they are published or not. The documents may come from teaching and research institutions in France or abroad, or from public or private research centers.
L'archive ouverte pluridisciplinaire HAL, est destinée au dépôt et à la diffusion de documents scientifiques de niveau recherche, publiés ou non, émanant des établissements d'enseignement et de recherche français ou étrangers, des laboratoires publics ou privés. 


\title{
Coherent illusory contours reduce microsaccade frequency
}

\author{
Alexis. D.J. Makin ${ }^{1,3}$, Rochelle Ackerley ${ }^{2,4}$, Kelly Wild ${ }^{2}$, Ellen Poliakoff ${ }^{1}$, Emma \\ Gowen ${ }^{2}$ and Wael-Deredy ${ }^{1}$
}

1: School of Psychological Sciences, University of Manchester, Oxford Road, Manchester, United Kingdom, M13 9PL

2: Faculty of Life Sciences, University of Manchester, Moffat Building, Sackville Street, Manchester, United Kingdom, M60 1QD

3: Department of Biological and Developmental Psychology, University of Liverpool, Liverpool, United Kingdom, L69 3BX

4: Sahlgrenska Academy, University of Gothenburg, Gothenburg, Sweden.

Corresponding Author: Dr Alexis D.J. Makin, Department of Biological and Developmental Psychology, University of Liverpool, Liverpool, United kingdom, L69 3BX, email: alexis.makin@liverpool.ac.uk, tel: 01517942000

\begin{abstract}
Synchronized high-frequency gamma band oscillations $(30-100 \mathrm{~Hz})$ are thought to mediate the binding of single visual features into whole-object representations. For example, induced gamma band oscillations (iGBR) have been recorded $\sim 280 \mathrm{~ms}$ after the onset of a coherent Kanizsa Triangle, but not after an incoherent equivalent shape. However, several recent studies have provided evidence that the EEG-recorded IGBR is a by-product of small saccadic eye movements (microsaccades). Considering these two previous findings, we hypothesised that there would be a more microsaccades following the onset of a coherent Kanizsa triangle. However, we found that microsaccade rebound rate significantly higher after an incoherent triangle was presented. This suggests that microsaccades are not a reliable indicator of perceptual binding. Moreover, the result also implies that iGBR cannot be universally produced by ocular artefacts.
\end{abstract}

\section{Keywords}

Gamma band oscillations, saccades, spike potential, Kanizsa triangles, EEG

\section{Introduction}

The brain is organized with discrete populations of neurons sensitive to particular dimensions of sensory input, such as orientation or velocity. In order to represent a complex, real-world object, the relevant information must be bound together, and irrelevant information arising from background objects or memory must be suppressed. Binding could be achieved through synchronization of high frequency gamma band oscillations ( $\sim 30$ to $100 \mathrm{~Hz}$ ) across the relevant neural populations (Schroder \& Lakatos, 2009). These gamma band oscillations can be recorded using a variety of methods, which differ in their spatial resolution. For example, comparable recordings have been obtained with invasive microelectrode techniques in monkeys and cats, and in the human electroencephalogram (EEG, Fries, Reynolds, Rorie, \& Desimone, 2001).

A stereotypical pattern of gamma band activity follows the presentation of a visual stimulus. First, there is the evoked gamma band response, which occurs around $100 \mathrm{~ms}$ after stimulus onset. This is time-locked to stimulus onset. Then, at around $300 \mathrm{~ms}$ post stimulus, the induced gamma band response (iGBR) becomes apparent. The onset of the iGBR varies from trial to trial and is of interest because it is modulated by a variety of cognitive factors. For example, Tallon-Baudry et al. (1996) 
found enhanced $30-40 \mathrm{~Hz}$ iGBR at around $280 \mathrm{~ms}$ after the onset of a coherent Kanizsa triangle, but not after an incoherent equivalent (Figure 1A). This activity could reflect the cognitive process required to link the three inducer shapes in order to perceive the illusory contour.

However, in 2008, Yuval-Greenberg et al. (2008) published a study that raised serious doubts about the validity of all EEG research into the iGBR. They replicated the general patterns of Tallon-Baudry et al. (1996), but found that iGBR was likely to be produced by muscle artefacts resulting from miniature saccadic eye movements. The muscle contractions associated with these microsaccades produce a spike potential in the EEG, which appears as a transient burst of broadband activity in the timefrequency domain. Yuval-Greenberg et al. (2008) argued that this artefact has frequently been mistaken for the iGBR. In support, they produced convincing correlational evidence. For example, they analyzed their data on a trial-by-trial basis, and found that microsaccades nearly always produced a transient burst of gamma band activity $\sim 4$ ms later. It was also found that the iGBR all but disappeared on trials where microsaccades were absent (see Yuval-Greenberg \& Deouell, 2011 for comparable results with auditory stimuli).

One implication of Yuval-Greenberg et al.'s work is that microsaccades are associated with perceptual binding of stimulus features. If previous EEG based iGBR recordings were inadvertently measuring microsaccades, and they found more iGBR in conditions where perceptual binding occurred, it can be inferred that that microsaccades must by involved in, or at least a by-product of, perceptual binding. The current study tested this hypothesis. We recorded eye position data while participants viewed a series of Kanizsa triangles or incoherent equivalents (Figure 1A). If microsaccades are associated with perceptual binding, then there should be more microsaccades for coherent Kanizsa triangles than incoherent triangles.

\section{Materials and Method}

\subsection{Participants}

Twenty-one healthy participants were involved (Age 18 to 42, 10 Female) who had normal or corrected-to-normal vision. Participants gave written informed consent and received $f 20$ compensation.

\subsection{Apparatus}

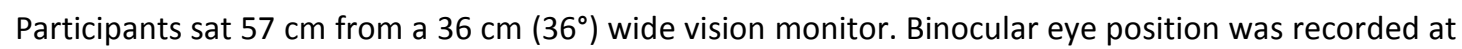
$500 \mathrm{~Hz}$ from an Eyelink II head mounted eye tracker, which was calibrated 3-4 times in each experiment. During calibration, participants following a cross as it moved $3.52^{\circ}$ horizontally back and forward across the centre of the screen. The cross remained static for 1 second at each position, and it changed positions 10 times. This was then repeated on the vertical axis.

\subsection{Design}

Stimuli were presented 60 times in each of 12 conditions $\left[2\right.$ size $\left(9^{\circ}, 18^{\circ}\right) \times 2$ coherence (coherent, incoherent) $\times 3$ duration $(50,100,200 \mathrm{~ms})]$, giving 720 experimental trials. The variation in triangle size and duration ensured that results were not unique to specific presentation parameters, and prevented participants from becoming over-familiar with the stimuli. The inducer shapes were $3.52^{\circ}$ in diameter, giving support ratios of approximately 0.2 and 0.4 for the large and small stimuli respectively. The fixation cross was equidistant from each point of the illusory triangle.

\subsection{Procedure}

Participants fixated on a central cross while viewing a randomized sequence of the stimuli shown in Figure $1 \mathrm{~A}$. In order to encourage participants to attend to the stimuli, they were given the task of 
counting rare oddball trials, in which one of the carrier shapes was grey instead of black. A total of 10 blocks were presented. In each block, there were 6 trials in each experimental condition, plus a variable number of oddballs (3-6), giving a total of 768 trials. The participants verbally reported the number of oddballs they had detected at the end of each block. An equal number of large, small, coherent and incoherent oddballs were presented, and the off-colour oddballs did not resemble one stimulus type in particular. Therefore we do not think that the oddball detection task would have produced differential microsaccade rates.

The duration between one triangle and the next was randomized between 1 and 1.5 seconds. After around 25 seconds had elapsed, participants were given a longer pause of around 10 seconds to rest their eyes. This happened 4-5 times per block. The experimenter restarted the experiment manually when the participant was ready to continue.

\subsection{Analysis}

Eye movements were analysed during a $1000 \mathrm{~ms}$ epoch, beginning $300 \mathrm{~ms}$ before triangle onset to $700 \mathrm{~ms}$ after triangle onset. Oddball trials were also excluded from analysis. Microsaccade latency, amplitude and peak velocity were obtained with an automated algorithm (Engbert \& Kliegl, 2003), which was identical to that used by Yuval-Greenberg et al. (2008). Briefly, eye position was transformed into velocity and an average velocity was computed over a moving window of 5 samples for each epoch. Microsaccades were defined where velocity exceeded 6 times the standard deviation of the velocity profile within the epoch, computed independently for horizontal and vertical components. Minimum duration of a microsaccade was set to 3 samples. The number of microsaccades occurring in sequential $50 \mathrm{~ms}$ time bins across this epoch was calculated, and this data was converted to microsaccade rate (microsaccades per second). Microsaccades were excluded from the analysis if they came from trials at beginning of a block or from a trial where blinks or high amplitude saccades $\left(>2^{\circ}\right.$ ) had occurred (648 cases, $6.25 \%$ ).

Microsaccade rate was then analyzed as a function of 4 within-participant factors using a repeated-measures ANOVA [Coherence (coherent, incoherent) $\times$ Size $\left(9,18^{\circ}\right) \times$ Duration $(50,100$ or $200 \mathrm{~ms}) \times$ Time (-300 to +700 in $50 \mathrm{~ms}$ time bins)]. The Greenhouse-Geisser correction factor was applied when the assumption of sphericity was broken. Many of the variables violated the normality assumption according the Kolmogorov-Smirnov test $(84.16 \%, p<0.05)$. Although ANOVA is considered to be valid under such circumstances, we used non-parametric post-hoc tests to ensure that the important patterns could not be attributed to unrepresentative data points.

\section{Results}

\subsection{Microsaccade characteristics}

The number of microsaccades varied greatly between participants. The maximum number of microsaccades produced by any participant in this experiment was $1530(\sim 2.13 \mathrm{~Hz})$, the median number was $292(\sim 0.4 \mathrm{~Hz})$ and the minimum number was $39(\sim 0.05 \mathrm{~Hz})$. In addition to microsaccade rate, we measured both amplitude and peak velocity of each individual microsaccade. After removing outliers where peak velocity was $>1500 \% \mathrm{~s}(<1 \%)$, these variables were positively correlated $(r=0.78$, $p<0.001$ ), indicating that they followed the saccadic main sequence (Bahill, Clark, \& Stark, 1975).

\subsection{Microsaccade rate}

Figure $1 \mathrm{~B}$ shows Grand-average microsaccade rate as a function of time from stimulus onset in each condition. It can be seen that microsaccade rate drops considerably at around 100-200 ms post stimulus, before peaking at 300-400 ms. This pattern was confirmed by a strong main effect of Time ( $F$ $(1.83,36.59)=12.755, p<0.001)$. In Figure $1 \mathrm{C}$, it can be seen that the peak was later when the stimuli was presented for the longest duration $(200 \mathrm{~ms})$ than when it was presented for 50 or $100 \mathrm{~ms}$. This produced a Time X Stimulus Duration interaction $(F(3.73,65.49)=7.311, p<0.001)$. A non- 
parametric Friedman's ANOVA confirmed that there was a difference between the three Duration conditions at both 325 and $425 \mathrm{~ms}$ after stimulus onset $(p<0.008$, vertical arrows, Figure $1 C)$. The omnibus ANOVA also produced a significant Time $X$ Coherence interaction $(F(7.31,146.26)=2.211, p$ $=0.034)$, and a non-parametric Wilcoxon Signed Ranks Test revealed that this resulted from a higher microsaccade rate in the Incoherent condition at $325 \mathrm{~ms}$ post stimulus ( $p=0.009$, Vertical arrow, Figure 1D). Peak rate was greater on incoherent trials in all duration and size conditions (Figure 1F). There was no Time $X$ Size interaction $(F(7.18,143.90)<1$. N.S., Figure $1 E)$, and no other main effects or interactions approached significance $(F(7.817,156.311)<1.486, p>0.167)$.
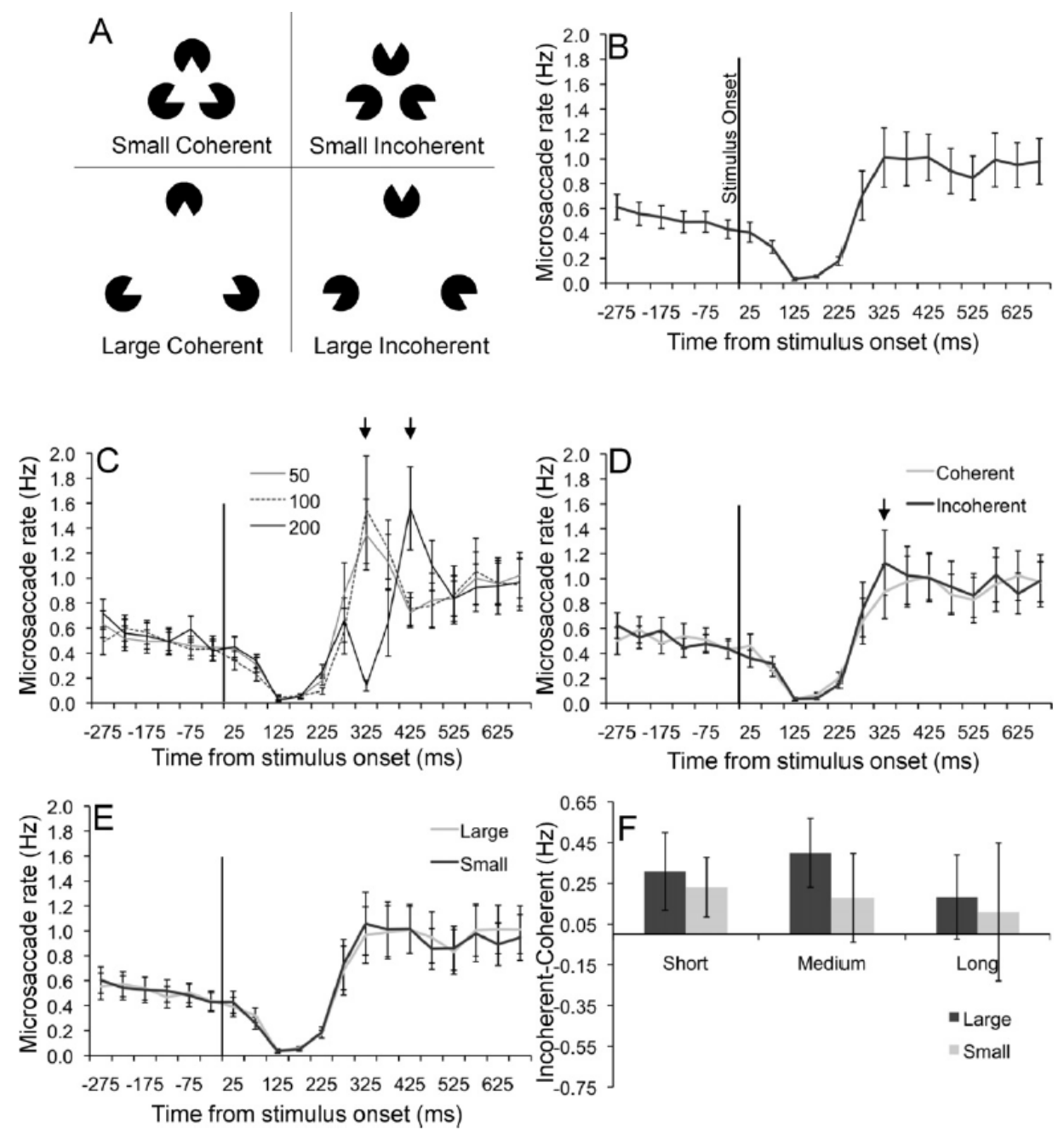

Figure 1: Stimulus and Results

A) The experimental Stimuli. Kanizsa Triangles are produced by the orientation of 3 black inducer shapes on a white background (coherent conditions). Reorienting the inducer shapes eliminated the illusory contour (incoherent conditions). Large and small stimuli were presented for either, 50, 100 or $200 \mathrm{~ms}$. B) Grand average microsaccade rate collapsed across all conditions. Microsaccade rate was calculated in 20 separate 50 millisecond time bins, and data is plotted as a function of Time from stimulus onset. C) The same data shown, but collapsed across the Coherence and Size factors only. D) The same data collapsed across Size and Duration factors. E) The same data collapsed across Coherence and Duration Factors. F) Comparison of grand-average microsaccade rate during the peak time bin between the Incoherent and Coherent trials, shown for each Duration and Size condition. Positive values in every condition indicate that microsaccade rate was universally higher in the incoherent trials (Error bars $=+/-1$ SEM). Vertical arrows in C and D highlight time bins discussed in the text. Vertical Lines indicate triangle onset. 
As mentioned in section 2.5, some microsaccades were excluded from the above analysis. The number of exclusions was modulated by Size, Duration and Coherence $\left(X^{2}>8.451, p<0.001\right)$. This effect, however, could not account for our main finding, since there were more microsaccades in the peak window of the incoherent conditions, even when no exclusion criteria were employed $\left(X^{2}=\right.$ $4.435, p=0.035)$.

\begin{tabular}{|c|c|c|c|c|c|}
\hline \multirow{2}{*}{} & \multicolumn{2}{|c|}{ COHERENT } & \multicolumn{2}{c|}{ INCOHERENT } & \multirow{2}{*}{ M } \\
\cline { 2 - 5 } & LARGE & SMALL & LARGE & SMALL & SM \\
\hline SHORT & 0.37 & 0.35 & 0.36 & 0.33 & 0.35 \\
\hline MEDIUM & 0.36 & 0.33 & 0.37 & 0.32 & 0.35 \\
\hline LONG & 0.40 & 0.37 & 0.35 & 0.40 & 0.38 \\
\hline M & 0.38 & 0.35 & 0.36 & 0.35 & \\
\hline
\end{tabular}

Table 1: Mean microsaccade amplitude in each condition.

Values are in degrees of visual angle. Marginal means are included in the row or columns labelled $M$.

\subsection{Microsaccade amplitude}

We measured microsaccade amplitude during the peak-rate time bin for each condition. There was little systematic difference between conditions (see marginal means, Table 1). In order to test whether there was a significant difference between the coherent and incoherent conditions, we sorted all trials by amplitude and conducted a median split. There were a near-equal number of coherent trials in high and low amplitude halves ( 251 vs. $265, X^{2}=0.380, p=0.538$ ). This implies that the coherence manipulation had no effect on microsaccade amplitude.

\section{Discussion}

This experiment measured the rate of sub-threshold saccadic eye movements while participants viewed coherent or incoherent Kanizsa triangles. Several known features of microsaccade rate were replicated here. First, amplitude and peak velocity were positively correlated (Abadi \& Gowen, 2004; Bahill et al., 1975). Second, there was a great deal of inter-individual variation in microsaccade rate (Abadi \& Gowen, 2004). Third, microsaccade rate dipped at around 100-200 ms after a visual onset, and then rebounded to reach a peak that was higher than the pre-stimulus baseline (Gowen, Abadi, Poliakoff, Hansen, \& Miall, 2007). The most important novel finding was that microsaccade rate was modulated by stimulus coherence: there were significantly more microsaccades in the rebound period when an incoherent triangle was presented ${ }^{1}$. This suggests that microsaccades are NOT an indicator of the brain's perceptual binding processes.

How, then, can this pattern be explained? It is likely that the three inducer shapes are perceived as separate peripheral objects in the incoherent conditions, but as part of a single, central triangle in the coherent conditions. Without fixation demands, the incoherent shapes may elicit more saccades away from fixation to the peripheral stimuli. The microsaccades we recorded here could be a trace of these initiated-but-inhibited eye movements to the periphery (cf. Gowen et al., 2007). This finding can be compared to recent work by Yuval-Greenberg et al. (2008), who found the iGBR in human EEG data was associated both with visual binding and microsaccades. Their work implies that microsaccades should be more abundant in conditions where binding occurs. However, this hypothesis was not confirmed by our experiment. In fact, we found that microsaccade rebound was significantly lower in conditions that promote perceptual binding.

Looked at another way, our results indirectly suggest that the iGBR produced by Kanizsa triangles is NOT an artefact of microsaccades. If microsaccades were entirely responsible for the iGBR, then we 
would expect them to be more frequent in the coherent triangle conditions, but we found the opposite pattern. It is also interesting that the coherent stimuli did not lead to higher amplitude microsaccades. Yuval-Greenberg et al. (2008) found that microsaccade amplitude was positively correlated with EEG-recorded iGBR amplitude. This implies that microsaccade amplitude should be higher for the coherent triangles where iGBR is greater, but we this was not the case in our experiment. It is important to note that our stimuli differed from the fragmented images used by Yuval-Greenberg et al. (2008), so our results are not a direct contradiction. Instead, both studies contribute to the wider debate about the iGBR.

This above reasoning adds one of the arguments articulated by Schwartzman and Kranczioch (2010). These authors pointed out that while the spike potential (produced by contraction of the ocular muscles) will translate into a broadband transient in the frequency domain, some recordings of the iGBR have a limited frequency range, ergo, they may not produced by spike potentials. For example, the iGBR associated with coherent Kanizsa triangles was found to be in the $30-40 \mathrm{~Hz}$ (Tallon-Baudry et al., 1996) implying that recordings were of neural origin. Other recent work has also suggested partial independence of iGBR and microsaccades: for example, Pope et al. (2009) found that the EEGrecorded iGBR was reduced, but not completely eliminated, when the eye muscles where paralyzed pharmacologically. Meanwhile, Nottage (2010) found that data cleaning techniques that remove spike potentials from the raw data do not eliminate the iGBR. It is also worth considering that artefacts produced by eye muscles should have a fixed scalp topography and frequency distribution, but both of these aspects of the iGBR response are dependent upon stimulus parameters (Schwartzman \& Kranczioch, 2010). Furthermore, it is known that gamma power varies with the phase of lower brain frequencies, again implying neural origins (Schroder \& Lakatos, 2009). On balance, it seems ocular artefacts may sometimes, but not always, produce the EEG-recorded iGBR. The current study also supports this conclusion, albeit indirectly.

The above discussion implicitly supports the interpretations of Tallon-Baudry et al. (1996). It is known, however, that event related potentials differentiate Kanizsa shapes from incoherent equivalents as early as 150 ms post stimuli, (e.g. Shpaner, Murray \& Foxe, 2009), while the iGBR begins around 280 ms. This chronology questions the specificity of the iGBR in illusory contour processing. We leave this issue for future consideration. The microsaccade rate recorded here was at the lower end of the 0.3$1 \mathrm{~Hz}$ ranges recorded in previous studies (Abadi \& Gowen, 2004). The microsaccade detection algorithm certainly missed a number of cases. However, extensive visual inspection of the raw data revealed that a high number of trials were completely free from microsaccades. This suggests that in future, EEG researchers should be able to use a high-resolution eye tracker in order to identify and exclude trials that are contaminated by microsaccades, and still have enough data left over for meaningful analysis. However, given the large individual variation in microsaccade frequency profiles, this approach could entail excluding participants with high microsaccade rates, which would introduce a systematic bias into the participant sample.

In conclusion, we observed an increased rate of microsaccades when participants observed incoherent compared to coherent Kanizsa stimuli. This suggests that microsaccades are not involved with perceptual binding, but covert visuospatial exploration of peripheral objects. Moreover, the results imply that the EEG recorded iGBR to Kanizsa triangles is not entirely attributable to ocular artefacts. If it were, then there would be more microsaccades following presentation of coherent stimulus, but we found more microsaccades following an incoherent stimulus. While establishing the role of microsaccades in EEG recording remains of paramount importance, the current work provides hope that the EEG is capable of recording high frequency brain activity, despite the problems associated with small eye movements. 


\section{References}

Abadi, R. V., \& Gowen, E. (2004). Characteristics of saccadic intrusions. Vision Research, 44, 26752690.

Bahill, A., Clark, M., \& Stark, L. (1975). The main sequence, a tool for studying human eye movement. Mathematical Biosciences, 24, 191-204.

Engbert, R., \& Kliegl, R. (2003). Microsaccades uncover the orientation of covert attention. Vision Research, 43, 1035-1045.

Fries, P., Reynolds, J. H., Rorie, A. E., \& Desimone, R. (2001). Modulation of oscillatory neuronal synchronization by selective visual attention. Science, 291, 1560-1563.

Gowen, E., Abadi, R. V., Poliakoff, E., Hansen, P. C., \& Miall, R. C. (2007). Modulation of saccadic intrusions by exogenous and endogenous attention. Brain Research, 1141, 154-167.

Nottage, J. (2010). Uncovering Gamma in Visual Tasks. Brain Topography, 23, 58-71.

Pope, K., Fitzgibbon, S., Lewis, T., Whitham, E., \& Willoughby, J. O. (2009). Relation of gamma oscillations in scalp recordings to muscular activity. Brain Topography, 22, 13-17.

Rolfs, M., Laubrock, J., \& Kliegl, R. (2006). Shortening and prolonging of saccade latencies following microsaccades. Experimental Brain Research, 169, 369-376.

Schpaner, M, Murray, M.M, \& Foxe, J.J. (2009). Early processing in the human lateral occipital complex is highly responsive to illusory contours but not salient regions. European Journal of Neuroscience, 30, 2018-2028.

Schroeder, C.E., \& Lakatos, P. (2009). The gamma oscillation: Master or slave? Brain Topography, 22, 24-26.

Schwartzman, D., \& Kranczioch, C. (2010). In the blink of an eye: The contribution of microsaccadic activity to the induced gamma band response. International Journal of psychophysiology, Article in press.

Tallon-Baudry, C., Bertrand, O., Delpuech, C., \& Pernier, J. (1996). Stimulus specificity of phase-locked and non-phase-locked $40 \mathrm{~Hz}$ visual responses in human. Journal of Neuroscience, 16, $4240-$ 4249.

Yuval-Greenberg, S., Tomer, O., Keren, A. S., Nelken, I., \& Deouell, L. Y. (2008). Transient induced Gamma-Band Response in EEG as a manifestation of miniature saccades. Neuron, 58, 429441.

Yuval-Greenberg, S \& Deouell, L. Y. (2011). Scalp-recorded induced gamma-band responses to auditory stimulation and its correlations with saccadic muscle activity. Brain Topography, 24, 30-39.

\section{Footnote 1}

There was also an effect of stimulus duration: the long $200 \mathrm{~ms}$ condition produced an atypical profile of microsaccade rate over time (Figure 1C). How can this be understood? We argue that visual processing, and not stimulus offset, produced the microsaccade rebound in the shorter conditions; it is otherwise difficult to explain why the rebound was affected by stimulus coherence and why the pattern is typical of the visually-induced microsaccade response. In the $200 \mathrm{~ms}$ condition, it is likely that a second period of suppression and rebound could result from visual offset (cf. Rolfs, Laubrock, \&

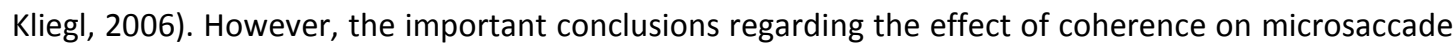
rate can be drawn from the 50 and 100 ms conditions alone.

\section{Acknowledgements}

The Economic and Social Sciences Research Council sponsored Alexis Makin, while the Medical Research Council sponsored Rochelle Ackerley and Kelly Wild. 